\title{
Tripartite Mechanism of Neural Memory: Proof-of-Concept with Neuromimetic Impedence Electrodes
}

Gerard Marx ${ }^{1 *}$, Chaim Gilon ${ }^{2}$

${ }^{1}$ Biotech Ltd., Jerusalem, Israel.

${ }^{2}$ Institute of Chemistry, Hebrew University Jerusalem, Israel.

*Corresponding Author: Gerard Marx, Biotech Ltd., Jerusalem, Israel. Received date: August 31, 2020; Accepted date: September 07, 2020; Published date: September 11, 2020

Citation: G Marx, C Gilon. (2020) Tripartite Mechanism of Neural Memory:Proof-of-Concept with Neuromimetic Impedance Electrodes.; Biomedical Research and Clinical Reviews. 1(3); DOI: 10.31579/2692-9406/021

Copyright:@2020 Gerard Marx, This is an open-access article distributed under the terms of the Creative Commons Attribution License, which permits unrestricted use, distribution, and reproduction in any medium, provided the original author and source are credited.

\begin{abstract}
The idea that neural signaling is the basis of mental processes has a long history. We graphically summarize salient developments in the neurobiology of signaling, as a Timeline. In particular, we review the "tripartite mechanism" of neural memory, which centers on the interactions between a neuron with its surrounding extracellular matrix (nECM) doped with metals and neurotransmitters (NTs). Essentially, the neuron employs the nECM as its "memory material", wherein it uses dopants to encode cognitive units of information (termed "cuinfo"). The NTs, which elicit bodily reactions (feelings), also encode past feelings as emotions, which "color" mental states in real-time and in memory.

In the interest of developing experimental tests of the tripartite mechanism, impedance glass electrodes were covalently coated with an exemplar NT (oxytocin) or a sulfated tetra-saccharide analog of the nECM, were constructed and tested. The two types of coated, neuro-mimetic electrodes, termed "neulectrodes", were capable of detecting metals, such as $\mathrm{Hg}^{+2}, \mathrm{~Pb}^{+2}, \mathrm{Cd}^{+2}$, $\mathrm{Cu}^{+2}$, and $\mathrm{Zn}^{+2}$ with very high selectivity and sensitivity. The "neulectrodes" demonstrated that the chemodynamic interactions of metal cations with NTs or nECM-saccharide analogues can translate into electrodynamic signals. They experimentally validate the concept of the tripartite mechanism that underlies the chemo-electric encoding of neural memory.

Keywords: neuromimetic electrode; impedence; chemodynamic sensing; trace metals; neurotransmitters; memory
\end{abstract}

\section{Background}

Neurons are chemo- and electro-dynamically linked cells that express a talent of mentation and somehow encode cognitive information as the basis for neural memory. But details are lacking regarding the physicality of the neural code and where the memory trace (engram) is located. Many presume that memory is somehow stored in the synaptic gaps between neurons (Cajal, 1911; Hebbs, 1949 ; Kandel et al, 2013; Cizeron et al, 2020), though this seems questionable from the point of view of persistence and theoretic credibility (Arshavsky 2006; Gallistel \& King 2009; Amit 2013; Marx \& Gilon, 2012-2020).

Some researchers developed nano- or microdevices to enable simultaneous, long-term, multi-site, intracellular electrical recordings from single or many neurons (Spira \& Hai, 2013). While they explored the electrophysiologic aspects of synaptic signaling using sensing electrodes, they did not address the issue of the mental states achieved by neurons. Other workers suggested that memory is stored in the nucleus (Kandel, 2001; Josselyn \& Frankland, 2015), though the kinetics of nuclear processes appear to be too slow and require high energy.
The computer metaphor for neural memory exerts a strong influence on the field of cognitive neurobiology. Many see a direct parallel between a computer and the brain (Turing, 1943; McCulloch \& Pitts, 1943; von Neumann, 1958; Arbib, 1987, 2000; Piccinini G. 2006; Giudolin et al, 2011).

"Computational systems are useful to describe brain processes mathematically".

- Giudolin et al, 2011

But the metaphor is incomplete. Aside from the quite different energy expenditures by a brain compared to a supercomputer (i.e. 20 Watts vs 250 megaWatts), it does not account for emotive states achieved by neural systems, for which there are no digital or electrodynamic equivalents.

The idea of neural signaling as the basis of mental processes has a long history beginning with Galvani through Golgi, Cajal, Hebb and Kandel, as we graphically summarize in the Timeline presented below (Figure $1)$. 


\section{Milestones in theTimeline of Neurobiology}

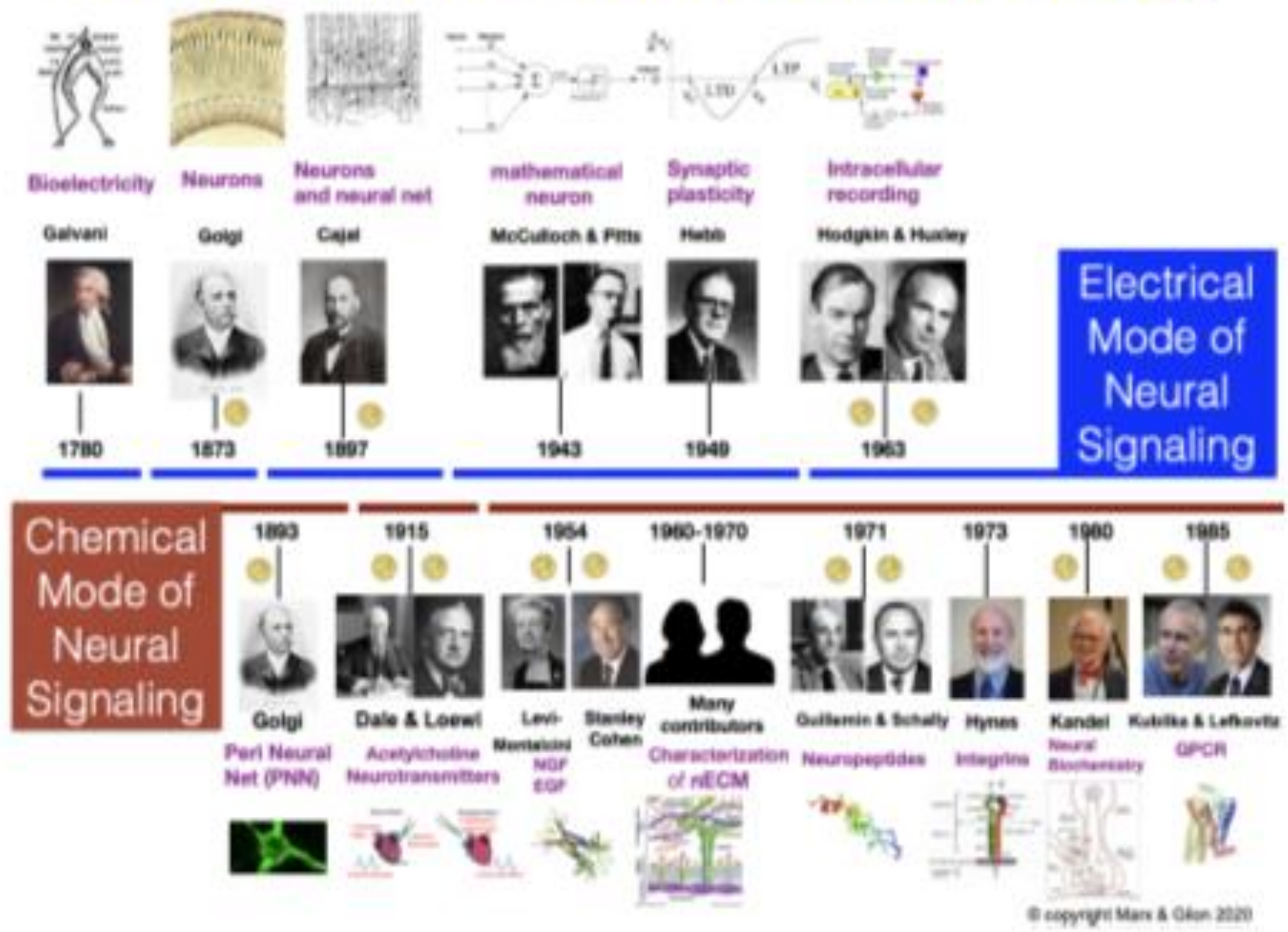

Figure 1. Milestones in the Timeline of neurobiology, acknowledging key contributors to unravelling the electrical and chemical modes of neural signaling. The Timeline (based on reviews: Kandel \& Squire, 2000; Langmoen \& Apuzzo, 2007; and our own work) emphasizes the parallel development of two dynamics of neural signaling: the electrical mode and the chemical mode.

Subsequent to the observations of Cajal of synaptic contacts between neurons (Cajal, 1911), a singular contribution to the electrical mode of was the McCulloch-Pitts mathematical model of a lone neuron (McCulloch \& Pitts, 1943). This approach was subsequently amplified by the concepts of electrical signaling expressed as "synaptic plasticity" and "long term potentiation".

The chemical mode took longer to develop, buttressed by the eventual confirmation of Golgi's observation of a perineural net (PNN), identified as a web of glycosaminoglycans (GAGs) around the neurons, now termed "nECM". The discovery of peptidic neurotransmitters (NTs) and their receptors also enabled a chemodynamic view of neural net communication. But signaling by itself does not resolve the core enigma of how neurons can remember existential events.

How is an emotive mental state encoded, stored and recalled from memory?

\subsection{Tripartite mechanism of neural memory}

We have proposed a tripartite mechanism for neural memory based on the formation of metal-centered complexes that attract neurotransmitters (NTs) (Marx \& Gilon, 2012-2019). Neurotransmitters (NTs) elicit psychic states parallel to physiologic reactions. Chemically, NTs bind to the metals anchored in the nECM. Effectively, they combine to establish the neuron's code for emotive states (Figure 2). 


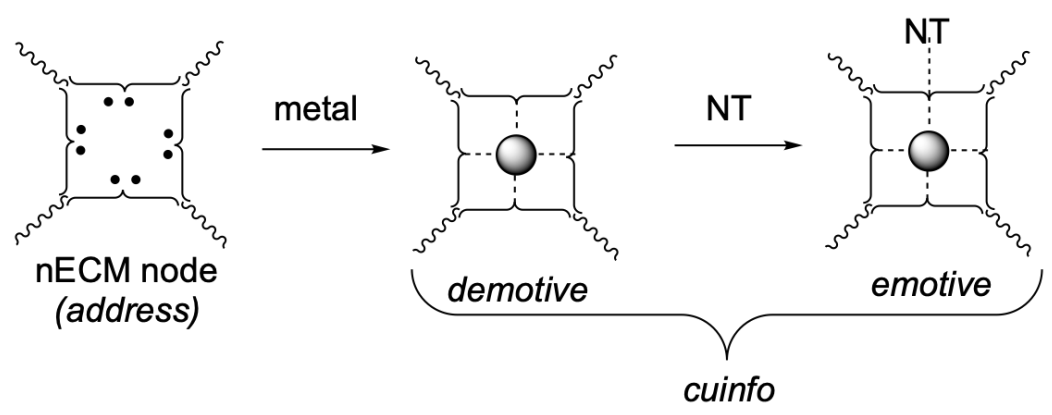

\begin{tabular}{|ll|} 
& $\begin{array}{l}\text { covalent lattice conectors in the } \\
\text { GAG building units of the nECM }\end{array}$ \\
$\cdots$ & $\begin{array}{l}\text { coordinate/chelator bond } \\
\text { ligands in the GAG building } \\
\text { units }\end{array}$ \\
mTnetal cation & neurotransmitter \\
\hline
\end{tabular}

Figure 2. Icons of an electron-rich nECM metal-confinement "address", capable of complexing with metals and neurotransmitters (NTs), thereby encoding a cognitive unit of information (cuinfo). Each NT endows the cuinfo (singular \& plural) with an emotive quality not available in binary code.

To encapsulate: The "neuron" marshals the components available to it, notably the surrounding extracellular matrix (nECM) and the dopants (metals and NTs) which the neuron accumulates within vesicles. The neuron employs these to encode cognitive units of information, called "cuinfo", metal-centered complexes within the nECM, described by a chemographic notation (Figure 2). This mechanism is universal, in that it applies to the recall function of all neural creatures, from C. elegans (302 neurons) to homo sapien ( $10^{15}$ neurons).

The term "chemotronic" refers to an intersection disciplines, such as those of chemistry with electronics or optics (see Khrustalev \& Rozhitskii, 2001). Here, we propose that memory links biochemical processes to psychological states (i.e. emotions). For example, hydrogels have been studied as structural entities, in terms of shear thinning, stretching, selfhealing and breaking strength. (Zhang \& Khadehosseini, 2017), but have not been studied for "chemotronic" signaling potential for encoding "psycho-chemical" states.

In this regard, consider the nECM as a 3-D hydrogel comprising a lattice of sulfated glycosamino-glycans (GAGs) (such as hyaluronate, chondroitins and heparans) which bind metal cations. The degree of sulfation of the GAG's is a major factor impinging on its metal binding affinity. Though a number of sulfation-enzymes have been identified (Gamma et al, 2006; Soares, 2016; Malaeb et al, 2019), correlation between the neuro-saccharide sulfation pattern and metal-binding characteristics remains obscure. Thus, experimental evaluation of the metal-binding affinities of specifically sulfated saccharides remains a goal of material scientists, which may incidentally clarify the signaling properties of neurons interacting with their nECM.

\section{Results and Discussion}

Following the idea of McCulloch \& Pitts (Figure 1) that described a single neutron in mathematical terms, we propose that the impedence electrode is a model for a chemo-electric neural receptor. Inspired by these ideas, our colleagues embarked on a program to fabricate various neuromimetic impedence electrodes ("neulectrodes") coated with materials available to the neuron, namely a neurotransmitter (oxytocin) or a sulfated tetrasaccharide analogue of the nECM. These electrodes could be tested for their binding affinity and selectivity to different metal cations.

We review two published examples that use Electrochemical Impedance Spectroscopy (EIS) as a method to detect changes in the electrochemical properties of coatings of "tripartite" components. We call such neuromimetic electrodes, "neulectrodes". EIS is highly sensitive to interactions of the electrode sensing layer with analytes, especially to conformational changes that result from metal binding to the surface. It does not depend on fluorescence, luminescence or light absorbance techniques frequently used to study metal binding by biopolymers.

\section{1. "Neulectrode" type 1: NT-coated impedance sensor}

An impedence electrode was modified in step-wise manner to covalently coat the sensing surface with oxytocin (OT), as schematically illustrated in Figure 3. 


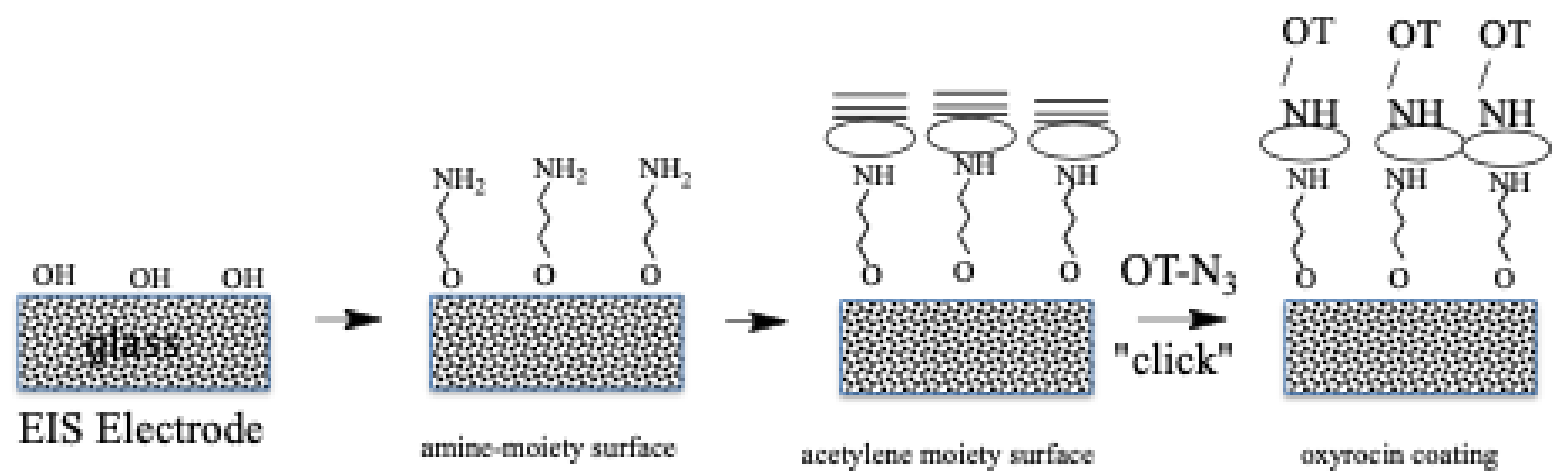

\section{OT- $\mathrm{N}_{3}=$ oxytocin-azide}

Figure 3. A schematic outline of the preparation of the oxytocin (OT) -coated EIS electrode. (The preparation details are described in Tadi et al, 2017).

The OT-coated electrode was particularly sensitive $\left(\sim 10^{-12} \mathrm{M}\right)$ to $\mathrm{Cu}^{+2}$ and $\mathrm{Zn}^{+2}$, but showed very low responsiveness to other metal cations $\left(\mathrm{Pb}^{+2}\right.$, $\mathrm{Mg}^{+2}, \mathrm{Cd}^{+2}, \mathrm{Ni}+2, \mathrm{Ca}^{+2}, \mathrm{Fe}^{+3}, \mathrm{Ag}^{+}$and $\mathrm{K}^{+}$) (Tadi et al, 2017). These results were in accord with other reports that oxytocin has great affinity for metals, which are cofactors in its physiologic effects (Neumann, 2007; Wyttenbach et al, 2008; van der Burg et al, 2009; Liu et al, 2005).

Each neural sensor has a unique specificity for a given NT ligand (Neumann, 2007). Thus, the OT-coated electrode, could be described as "neuro-mimetic" and might become diagnostically useful in a clinical setting to test for trace $\mathrm{Cu}^{+2}$ and $\mathrm{Zn}^{+2}$ in the circulating blood or lymph fluid of patients with multiple sclerosis, Alzheimer disorder, Parkinson disorder and autism.

\section{2. "Neulectrode" type 2: Sulfated oligo-Saccharide coated impedance sensor}

Uniquely sulfated tetra-saccharides were prepared (Table 1) and used to coat the EIS electrode
Surface to form the tetra-hyaluron glass, coated electrode (HA4-GCE) for testing for sensitivity to metal cations. (Alshanski et al. 2019) The hyluron tetra-saccharides (galactosamine N-Ac - Glucuronic acid; (GlcNAc$\mathrm{GlcA})_{2}$ ), were prepared as analogues of hyaluron (HA), a major polymeric component of the nECM (Rother et al, 2016; Yoon, 2016). While hyaluron is not generally sulfated, its tetra-saccharides serves as a good analogue of the generally sulfated chondroitins (glucosamine N-AcGlucuronic acid; GluNAc-GlcA $)_{n}(\mathrm{n} \geq 2)$. The process for covalently coating the electrode with the tetra-hyluron azides (Table 1) was similar to that developed for oxytocin (Figure 2), using tetra-hyluron azides instead of oxytocin azide (Alshanski et al, 2019).

\begin{tabular}{|c|c|c|}
\hline Code & sulfation pattern & structure \\
\hline HA4 & no sulfate & HA4 \\
\hline msHA4 & mono-sulfated & 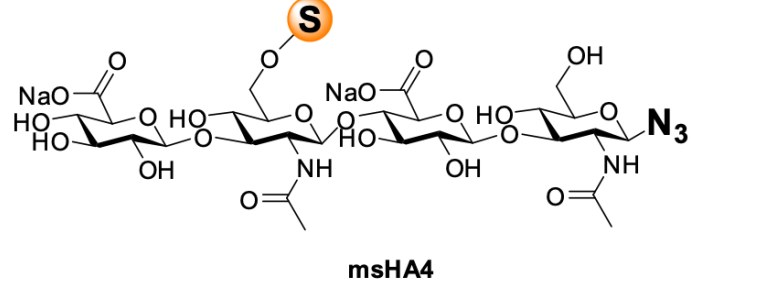 \\
\hline dsHA4 & di-sulfated $\left(6 \& 6^{\prime}\right)$ & 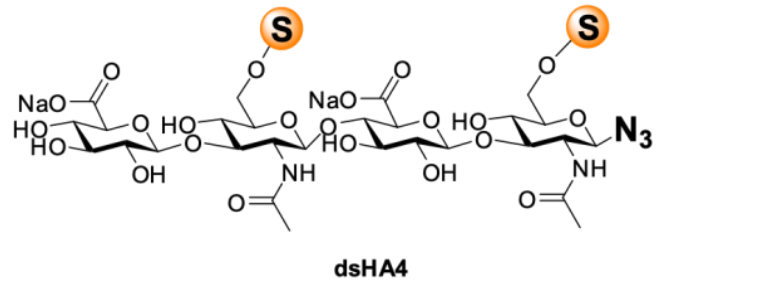 \\
\hline
\end{tabular}

Table 1: The sulfated tetra-saccharides used to coat the "neulectrode" type 2 (Alshanski et al. 2019) 


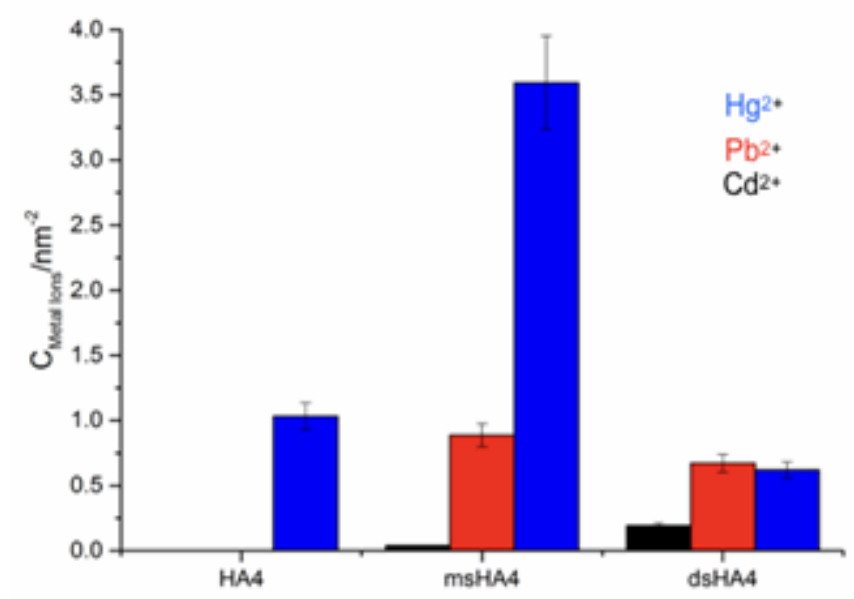

Figure 4. Response of different HA4-GCE to $100 \mathrm{nM}$ concentrations of $\mathrm{Cd} 2+, \mathrm{Pb} 2+$ and $\mathrm{Hg} 2+(\mathrm{N}=3)$. Black describes HA4-GCE, blue describes msHA4-GCE and red describes dsHA4-GCE (Alshanski et al, 2019).

The high sensitivity of the electrode coated with sulfated HA4-GCE to metals such as $\mathrm{Hg}^{+2}, \mathrm{~Pb}^{+2}$, and $\mathrm{Cd}^{+2}$ depended on the degree of sulfation, indicating that the interactions of sulfated oligo-saccharides with metal ions, especially heavy metal ones, are dictated by the sulfation patterns rather than by the core saccharide. Moreover, it corroborates our hypothesis that the sulfation pattern of the GAGs in the nECM determines the pattern of the metal distribution that is used for coding the neural memory. We hypothesized that NT (like oxytocin) could form ternary complexes with the metals embedded in the sulfated GAGs in the nECM, to be used for coding emotive memory. We also hypothesized that many thousands of sensors (i.e., GPCR, K channels, receptors, integrins), presented by the neural surface serve to "read" the cognitive information encoded by the metal -NT complexes in the $\mathrm{nECM}$ around the neurons.

\section{Conclusions}

The operational details of neural memory remain the focus of intense theoretical, modeling, basic and applied research. We have proposed that cognitive information received by the neuron is encoded as metal-centered complexes in the nECM surrounding the neurons. This implies that the neuron can form defined patterns of dopant complexes (metals and NTs) in the nECM. Moreover, it is capable of chemodynamic sensing such a pattern of metalcentered complexes and translating this information into electrodynamic signaling to the neural circuit (Figure 5).

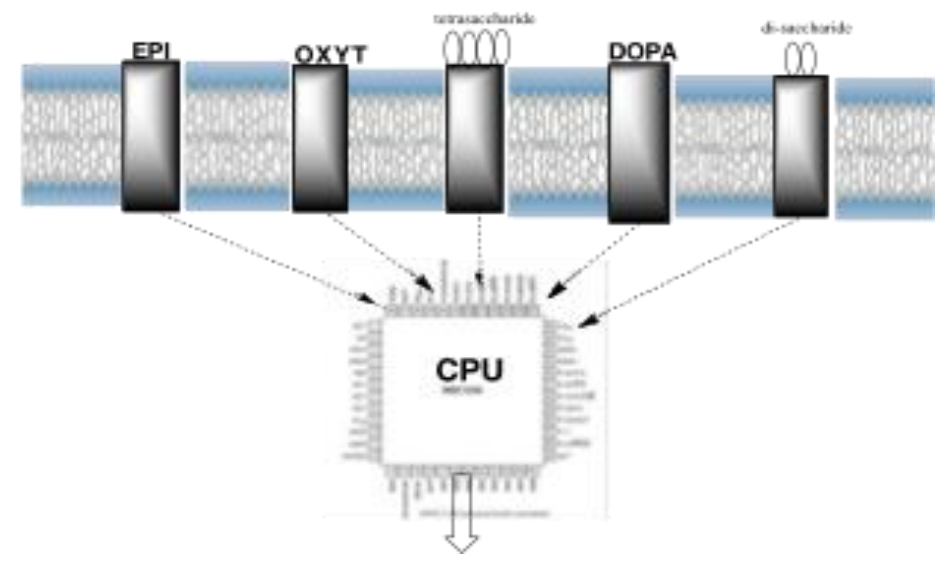

Figure 5. Conceptual model of a synthetic neuron with a variety of "neulectrodes" embedded in a lipid membrane. The sensory output of all would be directed to an electronic central processing circuit (CPU), permitting a brain-computer interface.

The "neulectrodes" are interesting from both fundamental and technological perspectives. They are truly neuro-mimetic in that they employ components available to the neuron. They may be useful as diagnostic tools or as implants for monitoring or controlling neural activity.

The two types of "neulectrodes" reviewed here demonstrate chemoelectric effects. Namely, that the chemo-dynamic interactions of metal cations with NTs or sulfated analogs of the nECM GAG can translate into electrodynamic signals. They experimentally validate the concept of the tripartite mechanism of neural memory.

\section{Acknowledgement}

(By GM). A memorium to my late wife and fan, the artist Georgette Batlle (1940-2009). Thanks to friends, Lilly Rivlin (New York, N.Y.) and the late Bill Needle (Eastchester, N.Y.) for their early encouragement and financial support in the period 1980-1984. 
We are pleased that we inspired and instigated this work by the research groups headed by Professor Shlomo Yitzchaik and Dr. Mattan Hurevich (Hebrew University) that fabricated and studied the coated electrodes, cited and discussed here.

\section{Conflict of Interest}

GM is a founder of MX Biotech Ltd., with the commercial goal to develop new classes of "memory materials" and devices.

CG is emeritus professor at the Institute of Chemistry, The Hebrew University of Jerusalem. He is active in developing technologies for the conversion of peptides and active regions of proteins into orally available drug leads.

This work did not receive any external funding support. Notwithstanding, the ideas forwarded here are scientifically genuine and presented in good faith, without commercial clouding of the concepts expressed herein.

\section{References}

1. Alshanski I, Blaszkiewicz J, Mervinetsky E, Rademann J, Yitzchaik S, Hurevich M. 2019.Sulfation patterns of saccharides and heavy metal ion binding.Chem. Eur. J. 25: 1 9

2. Amit, D. 2013 .Hebb vs Biochemistry: the Fundamentalist Viewpoint. In selected papers by Daniel Amit (1938-2007)

3. Arbib, M. A. 1987.Brains, Machines and Mathematics. 2nd Edition.Springer Verlag, New York.

4. Arbib, M. A. 2000.Warren McCullogh's search for the logic of the nervous system.Perspect. Biol. Med. 43, 193-216.

5. Arshavsky, Y. I. 2006 "The seven sins" of the Hebbian synapse: Can the hypothesis of synaptic plasticity explain longterm memory consolidation?Progress in Neurobiology 80, 99113.

6. Aschner, M. 2018.The functional significance of brain metallothioneins.FASEB J. 10, 1129-1136

7. Cajal R.Y. (1911).Histology of the Nervous System of Man and Vertebrates. Oxford University Press, 1995.

8. Cizeron, M, Qiu Z, Koniaris B, Gokhale R, Komiyama N, Fransén E, Grant S. 2020.A brainwide atlas of synapses across the mouse life span.Science 369(6501):270-275.

9. Gallistel, C. R., King, A. P. 2009.Memory and the Computational Brain.Wiley Blackwell, New York.

10. Gama C., Tully SE, Sotogaku N. , Rawat M., Vaidehi N., Goddard III W.A., Nishi A., Hsieh Wilson C. 2006.Sulfation patterns of glycosaminoglycans encode molecular recognition and activity.Nature Chemical Biology 2: 467-474.

11. Guidolin D., Albertin G., Guescini M., Fuxe K., Agnati L.F. 2011.Central nervous system and computation.Q. Rev. Biol. 86:265-285.

12. Josselyn S., Frankland P.W. 2015.Another twist in the histone memory code.Cell Research 25:151-152. doi:10.1038/cr.2014.134.

13. Kandel E. R., Squire L.R. (2000). Neuroscience: Breaking down scientific barriers to the study of brain and mind. Science 290: 1113-1120.

14. Eric R. Kandel E.R. 2001The Molecular Biology of Memory Storage: A Dialogue between Genes and Synapses Science 294: 1030-1038.

15. Khrustalev K.L., Rozhitskii M.M. 2001.Optochemotronics sensors-theory, experiment, practice.Proceedings of LFNM'2001. 3rd International Workshop on Laser and FiberOptical Networks Modeling, (Cat. No.01EX463).

16. Langmoen I.A., Apuzzo M.L. 2007.The brain on itself: Nobel laureates and the history of fundamental nervous system
function.Neurosurgery 61: 891-907; discussion 907-908.

17. Liu D., Seuthe A.B., Ehrler O.T., Zhang X., Wyttenbach T., Hsu J.F., Bowers M.T. 2005.

Oxytocin-receptor binding: Why divalent metals are essential.J. Am. Chem. Soc., 127: 2024-2025.

18. Malaeb W., Bahmad H.F., Abou-Kheir W., Mhanna R. 2019.The sulfation of biomimetic glycosaminoglycan substrates controls binding of growth factors and subsequent neural and glial cell growth.Biomater. Sci.

19. Marx G., Gilon C. 2013.The molecular basis of memory. MBM Part 2: The chemistry of the tripartite mechanism.ACS Chem. Neurosci. 4, 983-993, 2013.

20. Marx, G., Gilon C. 2016.The molecular basis of neural memory. MBM Part 6: Chemical coding of logical and emotive modes.Int. J. Neurology Res. 2: 259-268.

21. Marx G., Gilon C. 2017.The molecular basis of neural memory. MBM Part 7:Artificial intelligence (AI) versus neural intelligence (NI).AIMS Medical Science, 4: 254-273

22. Marx G., Gilon C. 2017.Molecular basis of neural memory. Part 8.Reviewing "neuro-mimetic" technologies.Neurosci. Biomed. Engin. 5.

23. Marx G., Gilon C. 2018. The molecular basis of neural memory. Part. 10. The sins and redemption of neurobiology.J. Neurology Neurocrit. Care 1: 1-7.

24. Marx G., Gilon C. 2019.The tripartite mechanism as the basis for a biochemical memory engram J. Integr. Neurosci. 18: 23 26.

25. Marx G, Gilon C. 2020.Quantum considerations of neural memory.In: Quantum Boundaries of Life, Volume 82In press. Elsevier, Amsterdam.

26. McCulloch, W. S., Pitts W. H. 1943.A logical calculus of the ideas immanent in nervous activity. Bulletin of Mathematical Biophysics 7, 115-133.Reprinted in: McCulloch W., Embodiments of Mind, MIT Press, 1965, pp. 16-39.

27. Neumann I.D. 2007.Oxytocin: the neuropeptide of love reveals some of its secrets.

Cell Metab. 5:231-233.

28. Piccinini G. 2006.Computational explanation in neuroscience. Synthese 153:343-353.

29. Rother S, et al. 2016Sulfated hyaluronan alters the interaction profile of TIMP-3 with the endocytic receptor LRP-1 clusters II and IV and increases the extracellular TIMP-3 level of human bone marrow stromal cells.Biomacromolecules 17:3252-3261.

30. Soares da Costa D., Reis R.I., Pashkuleva I. 2017.Sulfation of glycosaminoglycans and its implication in human health and disordersAnnu. Rev. Biomed. Eng. 19:1-26.

31. Spira M.E., Hai A. 2013.Multi-electrode array technologies for neuroscience and cardiology.Nature Nanotechnology 8: 83-95.

32. Tadi K.K., Alshanski I., Mervinetsky E., Marx G., Petrou P., Dimitrios K.M. Gilon C., Hurevich M., Yitzchaik S. 2017.Oxytocin-monolayer-based impedimetric biosensor for zinc and copper ions.ACS Omega 2: 8770-8778.

33. Turing, A. 1936. On computable numbers with an application to the Entscheidungs problem.Proc. London Math. SocSeries 2, 42: 230-265.

34. Von Neumann, J. 1958.The Computer and the Brain.Yale University Press, New Haven CT, 3rd Ed.

35. Van der Burg Y.E., Palmblad M., Dalebout H., Heeren R.M., Deelder A.M. 2009.Electron capture dissociation of peptide hormone changes upon opening of the tocin ring and complexation with transition metal cations.Rapid Commun Mass Spectrom. 23(1):31-38.

36. Wu et al. 2014.Structure of a class C GPCR metabotropic glutamate receptor 1 bound to an allosteric modulator.Science 
344: 58-64.

37. Wyttenbach T., Liu D., Bowers M.T. 2008.Interactions of the hormone oxytocin with divalent metal ions.J. Am. Chem. Soc., 130: 5993-6000.

38. Yoon, H.I. et al., 2016.Bioorthogonal copper-free "click" chemistry for labeling and tracking of chondrocytes in vivo.Bioconjug. Chem. 27: 927-936.

39. Zhang Y.S., Khadenhosseini A. 2017.Advances in engineering hydrogels.Science 356: 500.
This work is licensed under Creative Commons Attribution 4.0 License

To Submit Your Article Click Here: Submit Article

DOI: $10.31579 / 2692-9406 / 021$
Ready to submit your research? Choose Auctores and benefit from:

* fast, convenient online submission

* rigorous peer review by experienced research in your field

* rapid publication on acceptance

* authors retain copyrights

* unique DOI for all articles

* immediate, unrestricted online access

At Auctores, research is always in progress.

Learn more www.auctoresonline.org/journals/biomedical-researchand-clinical-reviews- 